\title{
LA RECEPCIÓN DE LA OBRA FREIREANA EN LA CIUDAD DE NUEVA YORK
}

\author{
THE RECEPTION OF THE FREIRE'S WORK IN NEW YORK CITY \\ A RECEPÇÃO DO TRABALHO DE FREIRE EM NOVA YORK
}

Revista do Programa de Pós-graduação em Educação da Unochapecó | ISSN 1984-1566 Universidade Comunitária da Região de Chapecó | Chapecó-SC, Brasil Como referenciar este artigo: CORCORAN, L. La Recepción de la Obra Freireana en la Ciudad de Nueva York. Revista Pedagógica, Chapecó, v. 21, p. 358-377, 2019. DOI: http://dx.doi.org/10.22196/rp.v22io.4880

RESUMEN: El artículo tiene dos objetivos. Primero, se rastrearála recepción dela obrafreireana enla Universidad de la ciudad de Nueva York (CUNY) durante los setenta y los ochenta, como fue traducido conceptualmente por Ira Shor. En este marco, se desarrollará el contexto en el cual Shor trabajaba y cómo Shor empezó a combinar la pedagogía de Freire con sus propios métodos para el desarrollo del pensamiento críticos de estudiantes. Segundo, se describirá la influencia actual de Freire en la pedagogía y en la sociolingüística. Se mostrará que la filosofía freireana todavía tiene un gran influencia en las prácticas pedagógicas de los educadores críticos en los Estados Unidos y que ha provisto un vocabulario teórico para seguir descubriendo cuestiones politicas e ideologicas y en el aula a través de la investigación del lenguaje cotidiano que estudiantes llevan consigo a la universidad.

Palabras clave: Pedagogía crítica. Ira Shor. Freire. Translingüismo.

ABSTRACT: This article has two objectives. First, it will follow the reception of Freire's work in the City University of New York (CUNY) during the seventies and the eighties, as it was translated conceptually by Ira Shor. In this framework, the context in which Shor worked will be developed, along with how Shor began to combine Freire's pedagogy with his own methods for developing the critical thinking of students. Second, the current influence of Freire will be described in pedagogy and in sociolinguistics. It will be shown that Freire's philosophy still greatly influences the pedagogical practices of critical educators in the United States and that it has provided a theoretical vocabulary in order to continue to discover political and ideological questions in the classroom through the investigation of the everyday language that students bring with them to the university.

Keywords: Critical Pedagogy. Ira Shor. Freire. Translingualism.

RESUMO: Este artigo tem dois objetivos. Primeiro, seguirá a recepção do trabalho de Freire na Universidade da Cidade de Nova York (CUNY durante os anos setenta e oitenta, como foi traduzido conceitualmente por Ira Shor. Neste contexto, o contexto no qual Shor trabalhou será desenvolvido, junto como Shor começou a combinar a pedagogia de Freire com seus próprios métodos para desenvolver o pensamento crítico dos alunos.Em segundo lugar, a atual influência de Freire será descrita na pedagogia e na sociolinguística.É demonstrado que a filosofia de Freire ainda influencia fortemente as práticas pedagógicas de Freire. educadores críticos nos Estados Unidos e que forneceu um vocabulário teórico para continuar a descobrir questões políticas e ideológicas em sala de aula através da investigação da linguagem cotidiana que os alunos trazem consigo para a universidade.

Palavras-chave: Pedagogia Crítica. Ira Shor. Freire. Translingualismo. 


\section{El contexto de recepción de la obra freireana en la ciudad de Nueva York}

La Universidad de la ciudad de Nueva York (CUNY) es un sistema universitario que actualmente incluye veinticinco instituciones de la educación superior. La primera universidad de CUNY fue fundada en 1847 por Townsend Harris, un político neoyorquino, quien fue también el primer embajador estadounidense en Japón, con el fin de "educar a todo el pueblo". Originalmente llamada "The Free Academy", esta universidad ahora se denomina "The City College of New York", y forma gran parte de la historia radical e izquierdista de la ciudad de Nueva York. City College, durante mediados del siglo XX, fue conocido como "el Harvard del proletariado", y todavía tiene la mayor cantidad de ganadores del Premio Nobel de todas las universidades públicas en los Estados Unidos. Durante aquella época también, especialmente durantes los sesenta, City College fue testigo de muchas manifestaciones estudiantiles por parte de la población afroamericana y puertorriqueña, demandando más acceso a la educación superior. En respuesta a estas manifestaciones, la administración de CUNY estableció una política de "admisiones abiertas" en los setenta, por la cual, cualquier egresado de una escuela secundaria de la ciudad de Nueva York podía inscribirse en CUNY gratis. También, durante esta ola de progreso, el programa SEEK fue fundado en CUNY con la meta de ayudarles a los estudiantes más marginados a tener éxito en sus estudios (Fullinwinder \& Lichtenberg, 2004, p. 61-62) . CUNY ya no es gratis, y aunque el programa SEEK todavía existe, la universidad (como muchas) ha sido golpeada por la creciente ideología del neoliberalismo de los fines del siglo XX, la cual seguimos presenciando a principios del siglo XXI.

Como el trabajo de Freire en Brasil durante la misma época, CUNY durante el siglo XX representó un "experimento" en la pedagogía democrática. Tanto los profesores como los estudiantes de CUNY, estaban pensando en nuevos paradigmas educativos, sociales y políticos. Los alquileres en la ciudad de Nueva York eran más baratos y los estudiantes inspirados por los movimientos de protesta contra la guerra de Vietnam y por el movimiento de los derechos civiles, entre otras movilizaciones políticas, veían la universidad como una bastión del radicalismo. En este entorno, la pedagogía freireana se arraigó en CUNY mediante la enseñanza y la teoría de Ira Shor. Actualmente, mientras la calidad de vida sigue empeorando para la clase obrera y la clase media en la ciudad Nueva York y mientras la ideología neoliberal no para de convertir a la universidad en un mero centro comercial donde los estudiantes compran una capacidad aislada una por una, la importancia de la pedagogía crítica también se ha reducido. Sin embargo, como sostiene Foucault (1977): "Donde hay poder, hay
* The Graduate Center, the City University of New York. Estados Unidos da América do Norte.

E-mail: lcorcoran@gradcenter.cuny.edu 
resistencia, y no obstante (o mejor, por lo mismo), esta nunca está en posición de exterioridad respecto del poder" (p. 116). Es decir que, aunque CUNY se haya alejado de su posicionamiento de mediados del siglo XX, todavía se van inventando nuevas formas de implementar la pedagogía crítica y de interpretar la obra freireana.

Este ensayo empieza por revisar la obra shoreana, y por contextualizarla en el entorno donde él empezó a combinar los métodos de Freire, y los cuales estaba desarrollando por su propia cuenta. Después se articula cómo la pedagogía crítica ha influido en nuevos abordajes en la sociolingüística, los cuales han tratado de mostrar que cualquier pedagogía que tenga que ver con la enseñanza del lenguaje, la escritura o la alfabetización, no puede evitar cuestiones políticas e ideológicas. El objetivo de este ensayo es demostrar que el axioma freireano, en el cual "leer la palabra es siempre leer el mundo", sigue teniendo un impacto en la pedagogía y en las ciencias sociales en los Estados Unidos.

Para entender el contexto en el cual Freire fue primero traducido conceptualmente en Nueva York es necesario también entender el campo académico en el cual fue recibido. En los Estados Unidos, los estudiantes universitarios en casi todas las universidades en el país, sean públicas o sean privadas, tienen que tomar un conjunto de cursos de la educación general. La justificación de tal programa de estudio es doble: por un lado, muchas veces estos cursos se tratan de materias de humanidades, por ejemplo, para que un estudiante de ingeniera pueda desarrollarse en lo "humano" y tener contacto con las artes y la literatura. Por otro lado, estos cursos, frecuentemente, se tratan de mejorar las habilidades "básicas" de los estudiantes, tanto en cuanto a la escritura y la lectura, como también en las matemáticas y las ciencias. Sin embargo, desde hace mucho tiempo, es casi universal que los estudiantes universitarios del primer año tomen una clase de escritura, ya que se supone que a los estudiantes les falta la capacidad lingüística necesaria para cumplir con los requisitos discursivos de la universidad. En los Estados Unidos, estos cursos se llaman "composición del primer año", y constituyen una enorme parte de los cursos ofrecidos por las universidades estadounidenses. Aunque están generalizados a través del país, el contenido de cada curso depende mucho de cada universidad. En universidades elitistas y privadas, por ejemplo, los cursos de composición del primer año suelen tratar la escritura creativa y artística, destacando la literatura como un referente para seguir. Al contrario, en las universidades públicas con una población de estudiantes que provienen más de la clase obrera, estos cursos se suelen enfocar en la gramática y las supuestas reglas lingüísticas, convirtiendo a la escritura en un juego de errores y correcciones.

De este modo, estos cursos, frecuentemente, han servido como un método de controlar los tipos de prácticas 
lingüísticas que están permitidas en la universidad. Paul Kei Matsuda (2006) sostiene que "el curso de composición del primer año ha sido un sitio de contención lingüística, poniendo en cuarentena a los alumnos que no se hayan adaptado a las prácticas lingüísticas dominantes por separarlos del resto de la población estudiantil" [traducción del autor] (Matsuda, 2006, p. 641). Se puede pensar en estos cursos metafóricamente como si fuesen una vacuna contra una enfermedad lingüística. Primero, los estudiantes reciben una inyección de un idioma "correcto" y, supuestamente, "neutro", antes de que estén autorizados a progresar en sus carreras universitarias e ingresar en los cursos más avanzados. En este esquema educativo, se puede decir que el lenguaje y el razonamiento son dos cosas distintas: uno tiene que aprender a escribir antes de aprender una materia especializada. Este modelo da por sentado que la escritura, o un idioma en general, es algo estable y determinado. También se nota que está en contra del principio fundamental de la pedagogía crítica: que la palabra no se puede leer sin leer a la vez al mundo. Es decir, en las universidades estadounidenses en el siglo $\mathrm{XX}$, una ideología se estaba desarrollando y separando la capacidad de escribir "correctamente", de la capacidad de pensar críticamente. Esta bifurcación sostiene que los alumnos ingresantes, primero tienen que aprender y después pueden empezar a teorizar. Semejante al contexto de Freire en Brasil, donde se suponía que la alfabetización se trata de simplemente transmitirles a los estudiantes estructuras lingüísticas sin debatir las condiciones de la alfabetización, en los Estados Unidos se supone que los estudiantes deberían aprender las herramientas "básicas" de la escritura sin analizar las condiciones materiales de la escritura.

Sin embargo, en los setenta y aún más en los ochenta, un grupo de profesores criticó este modelo seriamente, argumentando que no hay ninguna manera de separar el lenguaje de los papeles socioculturales que este juega. Es decir, afirmaron que es imposible extraer un idioma neutro de los varios actos de habla que componen los usos multidimensionales del lenguaje cotidiano. Si eso es cierto, no hay manera entonces, de primero enseñarles a estudiantes de los cursos de composición del primer año un idioma universal para que puedan después aplicar tal idioma en diversas situaciones. La premisa de que "el lenguaje es siempre ya ideológico" se volvió el lema de este grupo. James A. Berlin demuestra esta idea claramente, escribiendo que el lenguaje de la educación "nunca puede ser inocente, nunca puede ser el árbitro desinteresado de las ideologías de los demás, dado que siempre está sirviendo a alguna ideología u otra" [traducción del autor] ( Berlin, 1988, p. 477). Desde esta perspectiva, una pedagogía que solo pretende enseñar las estructuras "básicas" de la escritura sin cuestionar las ideologías contrapuestas que las componen, a la vez difunde su propia ideología. 
La construcción de lo neutro es un postura ideológica que quiere ocultar su propia ideología. Según este grupo de profesores, los cursos de composición del primer año ya implementan una ideología que articula la educación superior como solo el aprendizaje de las capacidades que tienen valor en el mercado laboral. Separar la enseñanza de la escritura, de cuestiones ideológicas, es tratar de posicionar a la universidad afuera de la política, una meta que la pedagogía freireana afirma como imposible.

En los ochenta, estos "cursos de la composición del primer año" que estuvieron por mucho tiempo considerados simplemente como unos cursos de servicio, como si fuesen semejante a enseñar a conducir, se convirtieron en el frente de batalla entre un ala pedagógica más conservadora y una más progresista. Los conservadores querían mantener la supuesta neutralidad de estos cursos, mientras que los progresistas querían reconocer la cuestión ideológica en el estudio de la escritura y el lenguaje. Además, esta batalla no solo consistía en cuál debería ser el último fin de este plan de estudio, sino también, en todo lo que tiene que ver con la enseñanza: métodos de calificar los trabajos de estudiantes, las actividades del aula, los textos para leer y, más importante, la relación entre el profesor y los alumnos. Revisando la literatura académica sobre este asunto, durante los setenta y ochenta, uno puede darse cuenta de la intensidad de la batalla. En las revistas académicas, colegas discutían amargamente el rol que tales cursos iban a jugar en la universidad en aquel entonces y en el futuro. Fue realmente un cambio en el paradigma acerca del estatus del lenguaje en la vida de la universidad. En la actualidad, esta batalla, por decirlo así, está todavía presente. Todavía gira en torno de si el trabajo de enseñar la escritura debería tratarse de aislar y transmitir un idioma supuestamente neutro, o centrarse en el desarrollo del pensamiento crítico a través del análisis de la ideología inherente al lenguaje cotidiano. Sin embargo, en la práctica, parece que la gran mayoría de las universidades estadounidenses todavía ponen mucho más énfasis en la pedagogía de transferencia y en la corrección de los supuestos errores lingüísticos de los estudiantes.

\section{La pedagogía de Ira Shor: los primeros libros}

Se puede considerar el trabajo de Ira Shor como el más importante en traducir conceptualmente la pedagogía freireana en los Estados Unidos y, más específicamente, en Nueva York. Shor fue contratado por CUNY en los setenta durante una ola de progresismo que estaba sucediendo, por lo menos, en el sistema universitario de educación pública en la ciudad de Nueva York. Como se ha dicho anteriormente, durante esta época, CUNY era gratis -aunque no lo es en la actualidad-, y no tenía requisitos de inscripción. Cada alumno que había cursado la escuela secundaria en Nueva York, se podía inscribir en 
la universidad, sin tomar exámenes de ingreso ni tener altas notas. En este sentido, CUNY era realmente una universidad para el pueblo neoyorquino, una medida diseñada para promover la movilidad social y para darle a la clase obrera una oportunidad para sumarse a la clase media y conseguir empleos profesionales. Como Shor suele describirlo, esta era una época más "democrática", porque la universidad dejaba de ser un método para mantener y justificar una jerarquía social y económica, y empezaba a ser un método para incluir a más gente de la comunidad local de educación superior. Sin embargo, había un "problema": de repente, la facultad de CUNY tenía que enseñar a una clase de alumnos que no estaban "preparados" para los desafíos y los discursos del mundo académico. Los "bárbaros" estaban en la puerta, y el primer instinto de muchos de la facultad de CUNY era reforzar las normas educativas tradicionales mediante una pedagogía que primero normalizaría a los alumnos por inculcarles las convenciones discursivas y las materias tradicionales de la universidad.

Aunque Shor no considerara el "problema" exactamente así, cuando empezaba a desarrollar su pedagogía crítica, está claro que sus primeros libros decían que este "problema" era algo esencialmente lingüístico. Los alumnos de la clase obrera neoyorquina tenían, y lo siguen teniendo, su propio "dialecto", un lenguaje que ellos mismos consideran algo poco formal: un idioma práctico para el trabajo o algo coloquial entre amigos y familia. Según esta forma de pensar, este lenguaje no sirve para reflexionar ni teorizar, sino solo para cumplir cosas en situaciones altamente prácticas y cotidianas: coordinar cosas laborales con colegas, hacer chistes, hablar de deportes, etcétera. Ver el lenguaje cotidiano de tal manera es verlo como si tuviese una correspondencia exacta entre cosa y palabra: el lenguaje, en este sentido, se usa para referirse al mundo de una manera "horizontal", sin la capacidad de criticar el mundo de una manera "vertical". Es decir, se supone que el lenguaje ordinario no tiene el poder necesario para ir más allá de la actividad práctica en el transcurso del día a día.

El primero paso de la pedagogía shoreana, entonces, es plantear la cuestión lingüística. Shor desafía la ideología ubicada que el pensamiento crítico y la habilidad de teorizar que requiere un lenguaje especializado que los profesores solamente pueden dominar. Shor intenta nivelar el discurso del profesor, poniéndolo en el mismo plano del de los estudiantes. Alejándose de la educación bancaria, Shor sostiene que el vocabulario y los contenidos educativos que posee el profesor no tienen un mayor valor de los que poseen los estudiantes. La pedagogía crítica, lejos de transmitir una materia concreta, ubica al profesor como un guía que iniciar diálogos mediante el planteo de problemas provenientes de la experiencia cotidiana. Esta es claramente una idea freirina. Freire aclara: "No sería posible llevar a cabo la educación problematizadora 
que rompe con los esquemas verticales característicos de la educación bancaria ni realizarse como práctica de la libertad, sin superar la contradicción entre el educador y los educandos" (p. Freie, 1970, p. 91). La diferencia entre el educador y los educandos está gravemente marcada por el curso de composición del primero año. Se supone que el profesor tiene todo el poder lingüístico y tiene el derecho indiscutible de juzgar el uso lingüístico de los estudiantes. El bolígrafo de tinta roja del profesor se transforma en un cetro en este entorno educativo. Por lo tanto, Shor implementa métodos que promueven el discurso de los estudios y reduce el discurso del profesor para equilibrar las diferencias lingüísticas inherente en tales cursos.

Para Shor, el lenguaje de sus estudiantes, aunque está estigimizado como deficiente o quebrado, ya contiene mundos de creencias, valores, roles sociales, formas de pensar y relaciones de poder. Por lo tanto, no hace falta que estudiantes de la clase obrera aprendan primero un lenguaje adecuado para pensar. Ellos pueden pensar y cuestionar, simplemente, por empezar a reflexionar sobre las herramientas lingüísticas que ya tienen a mano. De hecho, como una idea semejante de Freire, el lenguaje cotidiano, muchas veces, es un texto aún más rico para interpretar que los textos que ya se encuentran codificados como obras de literatura o los ensayos de escritores famosos. La pedagogía shoreana ubica a la vida diaria en su banalidad, normalidad, y transparencia, como el inicio, y muchas veces, el fin del desarrollo de pensamiento crítico de los estudiantes. Por otro lado, este abordaje también es político en dos sentidos. En primer sentido, tomar en serio la vida "normal" es una manera de incluir a la gente tradicionalmente excluida en los procesos educativos. En vez de solo darles los contenidos educativos de algunas materias para memorizar, los estudiantes son sujetos activos en la creación de nuevos conocimientos. En segundo sentido, y quizá el más importante, empezar con el lenguaje cotidiano de estudiantes provenientes de la clase obrera les permite analizar las estructuras de poder, sin requerir que ellos primero tengan que superar una barrera lingüística. Si la élite solo puede poseer un lenguaje capaz de reflexionar, la clase obrera nunca tendrá una oportunidad para desarrollar conciencia de su posicionamiento marginalizado.

Al contrario, cuando este lenguaje es empoderado por un educator crítico, ahora estos estudiantes tienen una herramienta para entender el mundo desde múltiples perspectivas, algunas de las cuales quizás los lleven a la acción política. Freire mantiene esta postura, ubicando a la educación como el acto central para cambiar las condiciones materiales del mundo: "La pedagogía del oprimido, aquella que debe ser elaborada con él y no para él, en tanto hombres o pueblos en la lucha permanente de recuperación de su humanidad. Pedagogía que hace de la opresión y sus causas, el objeto de reflexión de los 
oprimidos, de lo que resultará el compromiso necesario para su lucha por la liberación, en la cual esta pedagogía se hará y rehará" ( Freire, 1970, p. 42). Interpretando este planteamiento de Freire junto con el abordaje de Shor, está claro que la batalla política se lucha siempre en lo lingüístico. En muchos sentidos, el acto de recuperar la humanidad ocurre en la recuperación del lenguaje: que los significados de las palabras que se han quedado callados, vuelvan a hablarle al pueblo y tengan el poder conmovedor que es un requisito de la acción revolucionaria.

Ahí está la relación entre teoría y praxis que se suele encontrar en la obra freireana, en donde la educación se inicia con el mundo para teorizarlo y después para cambiarlo. Este hilo existencial siempre presente en la obra freireana, está también presente en la obra shoreana. La educación no es solamente un título ni un una actividad que suele ocurrir dentro de las paredes del aula, sino que es siempre, según Shor, un proceso de formación del sujeto. La relación entre aprender y enseñar, no solo se trata de lo que hay, sino también de lo que puede haber. Por lo tanto, el trabajo primario del educador crítico es ubicarse a sí mismo y ubicar a sus estudiantes dentro de esta apertura de lo que hay y lo que puede haber. Ahí también está el primer desafío de tal abordaje pedagógico, el cual es rearticular la educación para una población de estudiantes que por lo general han pasado por escuelas autoritarias que ponen el énfasis, casi sin excepción, en el mantenimiento de la jerarquía entre los alumnos y los docentes, y la transferencia de los hechos ya pasados desde el banco del maestro a los contenedores vacíos de los alumnos. Otra vez, según Freire, Shor describe esto como "el cambio de objetosujeto". Es decir, en la pedagogía crítica es necesario que los estudiantes dejen de ser meros objetos de los procesos educativos y que empiecen a ser sujetos en sí mismos en tales procesos.

La pedagogía shoreana demuestra la influencia freireana en su filosofía lingüística. Como dice Freire, que leer la palabra es siempre leer el mundo a la vez, la pedagogía shoreana intenta intervenir en el pensamiento de los estudiantes antes de llegar a la lectura de textos ya formalizados. Sin embargo, a diferencia de la situación de Freire, Shor desarrolla su método en un entorno institucional y burocrático que activa ciertas actitudes y comportamientos de resistencia de los estudiantes. Como en muchos lados, en los Estados Unidos, aunque no sea técnicamente así, ir a la universidad después de graduarse de la escuela secundaria, es casi obligatorio. Por lo menos, existe una norma social que dice que si uno quiere vivir el "sueño americano", necesita obtener un título universitario. Como resultado, muchos jóvenes se sienten frustrados después de pasar casi toda su infancia y adolescencia en la educación obligatoria, al enterarse de que tienen cuatro o cinco años más de la escuela solo para tener una oportunidad de competir en el mercado 
laboral, donde los trabajos calificados van desapareciendo. Además, cuando llegan a los pasillos y a las aulas de las universidades de CUNY, no pueden reconocer la diferencia entre esta nueva escuela y la última: las dos tienen el mismo formato, con paredes grises, escritorios estudiantiles incómodos, grandes cantidades de alumnos en cada clase y una pedagogía enfocada en corregir sus supuestamente constante errores. Ahí se marca una diferencia profunda con la situación de Freire. Este, por lo general, solía trabajar en contextos informales y no burocráticos, y con gente quien venía a estudiar voluntariamente.

También como un profesor de inglés, Shor tiene que enfrentar una narrativa ya internalizada por sus alumnos, que es la de poseer un idioma quebrado. La mejor estrategia que han aprendido tales estudiantes es quedarse callados y hacer las tareas requeridas para "sobrevivir" a la clase. Sin mucho esfuerzo, al contrario, el diálogo crítico en un contexto así, es casi imposible. Por lo tanto, en los ochenta, Shor empezó a experimentar con herramientas para arrancar el pensamiento crítico que evitaría activar una resistencia por parte de los estudiantes a su autoridad institucional como profesor. Muy semejante a las "codificaciones" de Freire, Shor suele usar los objetos más cotidianos del aula y las reglas de la universidad en sí, como catalizador de diálogo. Por ejemplo, Shor en $L a$ Educación que Empodera describe una situación que sucede el primer día de uno de sus cursos. Enfrentando un silencio profundo de un grupo de estudiantes a los que no les importa para nada el curso, Shor trata de arrancar una charla sobre el silencio en sí. Después de preguntarles a los alumnos, "¿qué está pasando aquí? Ni me conocen y ya no me quieren hablar". Un estudiante contesta: "el examen no es justo". Shor no sabe exactamente a qué se está refiriendo y responde: “¿qué examen?”.

"El examen de escritura”, dice el estudiante.

¿¿El que ustedes tuvieron que rendir para inscribirse en la universidad?".

"Sí".

“¿Qué tiene de malo el examen?”.

"No es justo", concluye el estudiante.

Y a partir de ahí la clase irrumpe en conversación [traducción del autor] (Shor, 1992, p. 3). En este momento, Shor logra encontrar un "tema generativo" que le permite a la clase debatir algo que le importa en un sentido más auténtico-existencial, que las estructuras "básicas" de la escritura. Sin embargo, esto no implica que la pedagogía shoreana no se enfoque en el desarrollo de la escritura de los estudiantes. La escritura se aprende a través del estudio de las condiciones materiales del sitio educativo. Este ejemplo demuestra cómo Shor traduce la práctica freireana de empezar con las experiencias reales de los estudiantes. El fin aquí no tiene nada que ver con dejar a los estudiantes 
quejarse de la escuela sin objetivo, sino de aislar un tema de diálogo que después los estudiantes y el profesor pueden seguir contextualizando y profundizando. Es decir, empezar con un hecho local le da a los estudiantes, en este caso de Shor, una oportunidad de reflexionar y de teorizar el mundo que ya los rodea.

Se encuentra un método similar en el primer libro del Shor, La enseñanza crítica y la vida cotidiana. En este libro, Shor representa una situación donde arranca un diálogo usando solamente los escritorios y las sillas del aula. Tomando las sillas de los estudiantes como objeto de análisis, Shor describe cómo una serie de preguntas sobre un objeto tan cotidiano pueden llevar al cuestionamiento de las estructuras de poder que se han vuelto invisibles en el aula. Después de pedirles a sus estudiantes que describan los componentes de sus sillas, que produce un listado de todos los elementos incómodos que tiene la silla, Shor empieza a hacerles preguntas:

Así que la silla es un objeto duro e inflexible para que sea difícil relajarse mientras uno se queda sentado. Pero ¿̇por qué no se permite que los alumnos se relajen en el aula? Porque nos quedamos dormidos, contestan los estudiantes. ¿̇Por qué se quedarán ustedes dormidos si la silla es cómoda? No se duermen en el cine o en casa cuando están en una silla cómoda. ¿Por qué se quedarán dormidos aquí? Porque el profesor es aburrido, contestan. Entonces, si el profesor es aburrido, eso hace que se duerman. ¿La respuesta es castigarlos a ustedes con una silla que los mantiene despiertos en vez de castigar al profesor por ser aburrido? ¿Por qué no cambiar al profesor para que la educación sea emocionante e inspiradora? ¿La gente se duerme cuando se le está ofreciendo algo que realmente quiere? [traducción del autor] (Shor, 1980, p. 160).

Estas preguntas revelan cómo puede ser posible iniciar un diálogo por tematizar las cosas dela vida cotidiana. Aunque una silla sencilla no es un gran texto escrito por un escritor literario, este objeto con las preguntas adecuadas puede llevar a los estudiantes al mismo análisis y a las mismas investigaciones que ocurrirían mediante la lectura de cualquier texto literario o filosófico. En este sentido, Shor es igual que Freire: no hace falta llevar textos oficiales al aula porque el mundo ya es un texto para leer. Lo que sí es necesario es la capacidad educativa de aislar y extraer temas del mundo cercano de tal manera que se puedan leer como si fuesen textos. Detrás de este método pedagógico, está la creencia de que la vida ya es estructurada por normas sociales, roles, jerarquías y relaciones de poder. Lo que es necesario es no crear textos para leer, sino darse cuenta de los textos que ya están presentes, pero se han vuelto invisibles. 


\section{Shor: "El tercer idioma"}

Loquedefine, porlogeneral, ala educación tradicional, es que ya tiene un conjunto de contenidos educativos que quieren que los estudiantes aprendan. Un curso de filosofía moderna tiene que enseñar las teorías de Descartes, Leibniz y Spinoza. Un curso de literatura estadounidense del siglo XIX tiene que enseñar Melville, Dickinson y Poe. En un marco limitado esto funciona bien: aprender en este sentido, significa dominar los contenidos educativos ya articulados por sus respectivas materias. Sin embargo, la alfabetización en el contexto de Freire y la escritura en el contexto de Shor, se ocupan con un meta educativa que va más allá del dominio de ciertas materias. En el caso de la pedagogía crítica, como se nota frecuentemente en la obra freireana y shoreana, la pedagogía se centra en el proceso de educación. Ahí está una distinción importante que separa dos cosas pedagógicas que se suelen confundir. Volviendo a los ejemplos anteriores, una cosa es enseñar la filosofía de la mente de Descartes y otra es plantear un problema y pedirles a estudiantes que desarrollen su propia filosofía de la mente. Es una cosa enseñar la concepción de la tragedia que Melville demuestra en su obra y otra es pedirles a estudiantes que desarrollen su propia teoría de lo trágico para examinar cualquier texto que se pueda encontrar en sus alrededores. La pedagogía crítica se deshace de contenidos específicos de la educación para centrarse en los procesos del pensamiento crítico. En esta concepción, el pensamiento crítico se desarrolla en conjunto con la alfabetización y la escritura. Sin embargo, es necesario reducir la magnitud de esta postura de la pedagogía crítica, dado que nunca es posible deshacerse totalmente del contenido; hablar es siempre hablar de algo. Pero se puede decir que tal postura de la pedagogía crítica sostiene que cualquier contenido puede servir para iniciar el pensamiento crítico. El "truco," para decirlo así, de la pedagogía shoreana, es la capacidad de usar los objetos, textos y vocabularios de la vida más cotidiana para empezar un diálogo en el aula.

Muy semejante al método socrático, la pedagogía crítica funciona para problematizar creencias comunes y para desarrollar una teoría a través del análisis de valores aparentemente obvios y normales. La clave aquí es que el diálogo no tiene ninguna orientación predeterminada ni ningún vocabulario necesario. Shor describe este proceso como el "tercer idioma" porque intenta crear un léxico de términos propios a cada curso, sin fijarse con antelación el lenguaje que se usará. "Se inventa de nuevo el tercer idioma en cada curso, situado en el lenguaje y en los niveles de desarrollo de los estudiantes, en la materia específica y en la política de la escuela, la universidad o la comunidad. No es un discurso estándar con solo una forma" [traducción del autor] (Shor, 1992, p. 256). El uso de "tercer" es para indicar que cada persona que va 
al aula (incluyendo el profesor) lleva consigo su manera idiosincrática de hablar y un punto de vista del mundo. El diálogo que ocurre en el aula es un intento por "superar" las limitaciones de cada respectiva subjetividad e inventar un lenguaje común propio en cada clase, y proviene de las condiciones existenciales compartidas. De tal manera, el tercer idioma es el vocabulario que tiene la potencia de vincular la teoría con la praxis en las condiciones donde se lleva adelante cada curso. Si cada acto político requiere un lenguaje para contextualizarlo y para que se lo lleve a cabo, el tercer idioma es el lenguaje creado, no solamente por el darse cuenta de las estructuras ideológicas que subyacen la vida cotidiana, sino también para el querer cambiarlas.

Así el tercer idioma es lo que resulta cuando el pensamiento crítico se va de lo ya se sabe y alcanza a descubrir nuevos conocimientos a través del intercambio de ideas y perspectivas en el aula. Vale decir que el diálogo se puede iniciar usando cualquier objeto, texto o creencia porque la experiencia ya está estructurada por ideologías contrapuestas. Como se dice anteriormente, hablar es siempre hablar de algo: "La participación de un hablante en los discursos presupone la suposición inconsciente de suposiciones básicas con respecto al ámbito de experiencia involucrada en el discurso" [traducción del autor] (Kögler, 1996, p. 207). Entonces, el tercer idioma es una herramienta para buscar de dónde son tales estructuras de la experiencia que se manifiestan en el lenguaje cotidiano. Esta idea es un eje central en la crítica de Freire contra la educación bancaria. Freire mantiene que el discurso que emerge del diálogo solo se puede lograr a través de un análisis honesto del lugar que tiene el sujeto dentro del mundo: "La reflexión que propone, por ser auténtica, no es sobre este hombre abstracto, ni sobre este mundo sin hombre, sino sobre los hombres en sus relaciones con el mundo. Relaciones en las que conciencia y mundo se dan simultáneamente. No existe conciencia antes y mundo después, y viceversa" (Freire, 1970, p. 94). Freire rechaza la posibilidad de dividir el sujeto, el mundo y el lenguaje en tres entes independientes. También aquí Freire muestra la influencia existencial de Sartre que tiene su obra. Según Sartre, lo que separa al ser humano de los otros entes del mundo es que el ser humano puede tomar una posición sobre su facticidad en este. Mientras que una piedra, por ejemplo, es definida totalmente por sus cualidades insuperables, una persona puede trabajar para superar su posicionamiento en el mundo a través del desarrollo de conciencia de él y una resolución para actuar sobre él. En este sentido, el tercer idioma que describe Shor es el comienzo de una conciencia existencial lograda mediante el diálogo.

La posibilidad de diálogo radica en el fenómeno que Pierre Bourdieu ha llamado el habitus. Este concepto interpreta que el mundo sociocultural es un campo de juego implícitamente estructurado por la ideología 
predominante. Ser sujeto, en este caso, es tener la capacidad tácita de manejarse en el habitus, cumpliendo con las "reglas" sin darse cuenta. El habitus como lo que articula al mundo ideológico sin necesidad de manifestar sus estructuras, formalmente posibilita las instituciones, los roles, las jerarquías, las interacciones y los encuentros de una cultura. Explicando este concepto de otra manera, se puede decir que el habitus es el "texto" que subyace cada acto de habla y comportamiento social que sujetos interpretan cuando hablan y actúan. Metafóricamente, este texto constituye el guión y los actores de los espacios normalizados por la ideología dominante (Bourdieu, 1980). Es por eso que es posible deshacerse de textos escritos en el marco de la pedagogía crítica porque el aula ya es un texto donde los estudiantes y los profesores están interpretando sus roles respectivos. El diálogo mediante su capacidad de plantear problemas y hacer que tales situaciones normalizadas se vuelvan extrañas, a la vez puede convertir este texto que se ha vuelto invisible en un texto visible y disponible para leer. Como Shor ha escrito, la meta de la pedagogía crítica como tal es "reexperimentar extraordinariamente lo ordinario" [traducción del autor] ( Shor, 1980, p. 97). El verbo "extrañar" sirve bien para explicar este concepto. Un diálogo en la pedagogía crítica trata de hacer que algo familiar, de repente, los extrañe a los estudiantes. A partir de ahí, el pensamiento crítico se desarrolla como un intento de entender lo que se ha vuelto extraño. Ni para Freire ni para Shor, la posibilidad de diálogo no se termina con el descubrimiento del texto que subyace la experiencia normalizada. El proceso que Freire llama la "humanización" del sujeto no descansa solamente en cuestionar este texto, sino se trata de trabajar para reescribirlo radicalmente. Por lo tanto, ser "humano" en este sentido, significa darse cuenta de que sí hay un texto normativo, pero nunca está terminado ni totalmente concretado. Uno puede asumir el poder de cambiarlo. Y así es la educación liberadora: la humanización de los estudiantes y los profesores juntos.

\section{La pedagogía freireana-shoreana en el principio del siglo XXI: el translingüismo}

La pedagogía shoreana ha tenido un fuerte impacto en la pedagogía de la escritura en la universidad. Sin embargo, el elemento político de este abordaje ha disminuido y otros pedagogos se han enfocado más en desarrollar métodos para centrar la experiencia de estudiantes sin considerar la meta freireana-shoreana. Esta se basa en provocar la conciencia de estudiantes para que se puedan analizar las condiciones materiales y después diseñar medidas de acción para cambiarlas. Es decir, es posible pensar la pedagogía dialogante como una práctica que puede servir para cualquier política educativa, sin fijarse necesariamente en entender el posicionamiento de las clases sociales dentro 
de las jerarquías socioculturales. En los Estados Unidos, frecuentemente, el término "pedagogía dialogante" ha terminado significando cualquier práctica educativa que tenga que ver con reducir el discurso del profesor en el aula y aumentar el de los estudiantes. En este sentido, el elemento radical de Freire y Shor se ha neutralizado y muchas veces se ha transformado en una pedagogía que se radica en la experiencia de estudiantes, sin contextualizar tal experiencia en las estructuras ideológicas de la cultura predominante.

Sin embargo, recientemente, el campo ha vuelto a pensar en las cuestiones políticas relacionadas con la pedagogía. El término "translingüismo" en la última década ha entrado en el léxico de la pedagogía de la escritura como un intento de rearticular las implicaciones políticas de la educación a través del cuestionamiento del lenguaje. El trabajo de sociolingüistas, como Ofelia García y Ricardo Otheguy, ha criticado severamente las concepciones tradicionales sobre el lenguaje y las fronteras que supuestamente existen entre los idiomas. Han denominado esta manera de separar los recursos lingüísticos de hablantes, siguiendo las fronteras de estados-naciones: la ideología "monolingual". En respuesta a esta ideología, han trabajando para desarrollar otra manera de concebir el lenguaje, usando el término "translingüismo" y "translanguaging". Partiendo de la teoría de los sociolingüistas de marco crítico, García y Otheguy han argumentado que los que suelen llamarse "distintos" idiomas, son realmente una construcción de poder colonial. Es decir, los entes que se llaman "inglés" y "español", por ejemplo, no se refieren a entes con realidad lingüística, sino a entes con realidad ideológica y política.

En la práctica, los hablantes "mezclan" con frecuencia "distintos" idiomas, utilizando una sintaxis híbrida sin ninguna falta de comprensión. Se usan comillas en las oraciones anteriores ya que, según estos autores, los términos como "mezclar" y "distinto idioma" solo tienen sentido refiriéndose al lenguaje dentro del marco de la ideología del colonialismo. En la práctica, los hablantes utilizan un repertorio lingüístico que no es marcado por fronteras nacionales, sino que es compuesto por un proceso de formación subjetivo-lingüístico que es transcultural y transnacional. Estos autores describen estas prácticas lingüísticas que van más allá o desafían las fronteras lingüísticas, con el término translanguaging, y sostienen que esto es "el uso del total repertorio lingüístico de un hablante sin prestar atención a las fronteras de idiomas definidas cultural y políticamente, que frecuentemente llevan nombres de estados o naciones" [traducción del autor] (p. Otheguy et al., 2015, p. 284). Este concepto plantea de nuevo que el lenguaje siempre es político y que la educación lingüística que trata de simplemente enseñar un idioma "neutro", ya está involucrado en una ideología nacionalista y colonial. Como muchos han afirmando, el 
control de lo neutro es donde el poder más está en juego. Concebir el lenguaje como una subjetividad lingüística ordenada por patrones complejos de poder, migración y política, en vez de concebirlo como un conjunto de distintos idiomas neutros y estándares, es desafiar que el aprendizaje puede ocurrir sin reconocer las ideologías contrapuestas inherentes en el uso más cotidiano del lenguaje.

Este abordaje translingüe, no solamente dirige la atención educativa a cómo las ideologías nacionalistas y colonialistas construyen un idioma "neutro," sino también a las condiciones materiales de donde emerge el lenguaje. Muchos investigadores en los Estados Unidos han estudiado cómo el racismo afecta profundamente a la educación lingüística y cómo el intento de enseñarles a los estudiantes un inglés "neutro", es también instalar un idioma que proviene de las clases más alta y, tradicionalmente, blancas de la sociedad estadounidense. Dicho de otra manera, enseñar el dialecto inglés que proviene de tal sector social, como si fuese estándar y neutro, perpetúa un acto de racismo lingüístico a través de un esquema educativo, aparentemente inocente.

Recientemente, los sociolingüistas Nelson Flores y Jonathan Rosa han sostenido que el cuerpo racializado contribuye más al significado de los actos de habla, que a las estructuras gramaticales que contienen los actos. Han denominado a este concepto como "ideologías raciallingüísticas", las cuales se utilizan para interpretar cómo hechos materiales y corporales condicionan cómo se percibe el lenguaje de estudiantes. "Sin un análisis de los códigos de poder, como una ideología racial-lingüística, no se puede cuestionar cómo la gente no racializada puede desviarse de prácticas lingüísticas idealizadas y disfrutar de la aceptación de las instituciones dominantes, mientras que la gente racializada puede adherir a tales prácticas y todavía encarar una exclusión institucional profunda" [traducción del autor] (Flores y Rosa, 2015, p. 165). Esta postura rechaza, otra vez, que es posible transmitir un idioma supuestamente neutro, sin tener en cuenta cuestiones ideológicas y materiales. Se nota que, aunque las personas racializadas dominen las prácticas extraídas de la clase dominante, todavía son excluidas de la cultura dominante por el hecho de que su aspecto corporal no encaje bien en la concepción del sujeto-hablante ideal. Aquí vale repetir el axioma freireano que dice que leer la palabra es siempre leer el mundo a la vez. Intentar evitar cuestiones ideológicas en el aula no se puede lograr porque este intento ya es una postura ideológica. Por ende, iniciar diálogos donde se debaten las estructuras ideológicas de la experiencia diaria y del lenguaje cotidiano, no es de ninguna manera politizar la educación, sino es una forma de reconocer el hecho de que la educación ya está politizada. Una pedagogía status quo trata de tapar este hecho para construir un conjunto de materias educativas y prácticas lingüísticas hipotéticamente neutras $\mathrm{y}$, de este modo, 
también trata de excluir a los estudiantes de los procesos educativos. Por otro lado, la pedagogía crítica intenta descubrir el mundo ideológico e incluir a los alumnos en el análisis de él. Aunque no aparezcan ningunas citas directas de Freire en el trabajo de estos pensadores, se siente su influencia. El planteamiento inicial de Freire de que la educación siempre involucra a la política, les provee un marco teórico dentro del cual, nuevas maneras de investigar la política inherente en la educación se pueden desarrollar.

\section{El contexto actual de la pedagogía crítica en la CUNY}

La teoría translingual orienta la educación hacia las condiciones materiales de donde emergen los actos de habla y para donde van. Una orientación así es importante porque en la ciudad de Nueva York existe una población de estudiantes definida, por lo que se la ha llamado "la superdiversidad" (Vertovec, 2007). Estos estudiantes representan patrones de migración, diferencias culturales, raciales y lingüísticas, y complejas relaciones con el mercado laboral. Por lo general, provienen de lugares que Mary Louis Pratt ha denominado "zonas de contacto", definiendo el término como los espacios geopolíticos donde "las culturas se encuentran, se enfrentan y se pelean, muchas veces en el contexto de relaciones de poder altamente asimétrico, como por ejemplo, el colonialismo o la esclavitud, o donde todavía se los sienten mediante sus consecuencias posteriores, provocando que la gente los siga experimentando en la actualidad" [traducción del autor] (Pratt, 1991, p. 34). Para muchos estudiantes de CUNY viniendo del Caribe, África y del sur de Asia, este término les es útil para empezar a entender los recurso lingüísticos que los estudiantes llevan consigo al aula, y también para descubrir que una ideología que fija idiomas nacionales como entes lingüísticamente reales, no funciona con respecto a tal población.

Por ejemplo, los estudiante de la CUNY provenientes de la República Dominicana, frecuentemente, poseen un conjunto de recursos lingüísticos que incluye elementos ideológicamente marcados, como inglés y otros como español. En los actos de habla diarios de estos estudiantes, no les importa si una palabra es "inglés" o "español". Lo suyo es otro idioma que trasciende fronteras nacionales por el hecho geográfico de donde está ubicada la República Dominicana y porque viven en comunidades translingües y transculturales en Nueva York. La perspectiva nacionalistacolonialista o monolingüe, no puede ver estas prácticas lingüísticas sin designarlas como lenguaje quebrado o una mezcla lingüística que lleva la connotación de algo inferior. Cuando esta población entra a la universidad, por lo general, la primera reacción de los profesores que les tienen que enseñar la escritura, es transmitir un inglés "neutro" que 
se puede usar como si fuese una herramienta básica. Los perfiles lingüísticos de tales estudiantes desencadenan una pedagogía enfocada en la corrección de errores lingüísticos y la construcción de un idioma ficcional que los estudiantes tienen que aprender.

La construcción y la transferencia de un idioma supuestamente neutro tiende a borrar los conocimientos lingüísticos-culturales que ya tienen los estudiantes. Como sostiene Freire, transferirles los contenidos educativos a los estudiantes, en vez de crearlos con ellos, inevitablemente convertirán a los educados en contenedores vacíos -una tabula rasa- sin experiencias anteriores ni un mundo fuera de las paredes del aula. Sin embargo, en la última década, una nueva ola de pedagogos ha tratado de implementar la pedagogía crítica mediante la teoría translingüe. Por ejemplo, Lucas Corcoran describe un contexto educativo en la actualidad que se parece notablemente al de Shor en los setenta y los ochenta. Corcoran aquí, teoriza la pedagogía con respecto a una población estudiantil donde casi cien por ciento de los alumnos diariamente usan recursos lingüísticos que no vienen del inglés. Trabajando dentro del programa SEEK del estado de la ciudad de Nueva York, diseñado para darle ayuda a la gente que tradicionalmente no ha tenido acceso a la educación superior por razones económicas, Corcoran se encuentra con alumnos que han internalizado la narrativa tóxica de que su lenguaje está quebrado. En el primer año de la universidad, los estudiantes de este programa, más que nada esperan lo mismo que han experimentado en la secundaria: que el profesor les dé tareas de escritura y después les corrija sus errores (Corcoran, 2017).

Corcoran desafía esta tendencia pedagógica para ubicar la investigación lingüístico-etnográfica de los propios actos de habla de los estudiantes, como el eje central del curso. Primero, en el curso que él delinea, los estudiantes juntan datos lingüísticos-etnográficos para escribir sobre sus propias experiencias con el uso del lenguaje y para entrevistar a un miembro de sus comunidades sobre sus experiencias lingüísticas. A partir de ahí, los estudiantes empiezan a teorizar estos datos mediante prácticas dialogantes; después investigan los temas, que descubren por leer textos sobre la lingüística que los ayuda a entender sus propias experiencias lingüísticas de otras perspectivas. La clave aquí es desarrollar la escritura de estudiantes sin ignorar el hecho de que ya tienen conocimientos lingüísticos avanzados, que forman parte de un mundo que también se merece ser reconocido por la institución educativa. De una forma paralela a Freire, quien trabajó en alfabetizar a estudiantes empezando con palabras e imágenes de las propias experiencias de la gente, este abordaje se fundamenta en las destrezas lingüísticas de estudiantes, y de ahí trata de agregar unas nuevas destrezas a las cuales ya pueden utilizar. 
Esta pedagogía translingüe con raíces freireanas afirma una postura de la sociolingüística crítica que es que el lenguaje nunca es solamente un saber estático, sino que es mejor considerarlo como una práctica: el lenguaje no es lo que un hablante sabe de las palabras, sino lo que hace con ellas. Alastair Pennycook aclara esta idea: "Una vez que empezamos a pensar en el lenguaje y a considerar el lenguaje como una práctica local y, por lo tanto, una actividad central que organiza la vida social que se ocurre en lugares específicos, un número de presuposiciones sobre el lenguaje no se puede sostener. La noción de que el lenguaje es un sistema, está desafiado por la noción de que el lenguaje es una acción" [traducción del autor] ( Pennycook, 2010, p. 2). Entender este punto es entender lo que Freire describe como la enfermedad de la narración que está presente en la pedagogía autoritaria. Solo un saber se puede transmitir como una serie de datos que se pueden escribir en libros y memorizar. $\mathrm{Al}$ contrario, el análisis de una práctica requiere otro abordaje que puede comprender sus cualidades existenciales, tanto en cuanto a lo ideológico, como en lo social. Ahí otra vez surge la pedagogía dialogante, ya que este método busca interpretar la experiencia sin convertirla en algo estático e inmóvil. El translingüismo entonces, se puede ver como una extensión de esta faceta de la pedagogía freireana, que se ha extendido a la sociolingüística y también a la pedagogía de escritura en las universidades estadounidenses.

\section{Conclusión}

Como en mucho lados, la universidad estadounidense está en crisis. La ola destructiva del neoliberalismo sigue reduciendo los recursos para la universidad pública, obligándola a aumentar la matrícula y forzando a los estudiantes a pedir préstamos estudiantiles para pagarla. Por otro lado, las universidades privadas en Nueva York pueden llegar a costar setenta mil dólares anualmente, excluyendo lo que los estudiantes tienen que pagar por libros, alojamiento y comida. Si uno camina por las calles de Nueva York, puede notar una alta cantidad de publicidades promocionando una universidad $\mathrm{u}$ otra, casi siempre destacando las ventajas que les darán a los estudiantes en el mercado capitalista-laboral. Al preguntarles a los estudiantes en el aula, “¿para qué estás aquí?”, se suele escuchar: "Para ganar dinero". No cabe duda de que esta respuesta es justa. En un mundo definido por competencia global, donde un pequeño porcentaje controla la gran mayoría de la riqueza y donde una ideología capitalistaindividualista va destruyendo lazos sociales convirtiendo vecinos en enemigos y colegas en competidores, considerar la educación superior como otra norma social que uno tiene que cumplir para sobrevivir, encaja en la lógica dominante.

El trabajo de la pedagogía freireana en Nueva York y en los Estados Unidos en la actualidad, es enfocarse en esta 
lógica dominante y cuestionarla. En la ciudad de Nueva York, viviendo en la capital de capitalismo, nos rodean los artefactos y las retóricas de la ideología neoliberal: es evidente que nos sobran textos cotidianos que podrían iniciar un diálogo crítico. En las tazas de café "to-go", en las publicidades del subterráneo y en las cafeterías se ven las estructuras que siguen formando la subjetividad neoliberal. Sin embargo, las herramientas de la pedagogía crítica siguen funcionando también. Es decir, aunque la ideología que enfrentamos a principios de siglo XXI haya cambiado, todavía la posibilidad de la humanización a través del diálogo es posible. De nuevo, el axioma freireano nos enseña que leer la palabra es siempre leer el mundo a la vez. El mundo está siempre ahí y siempre constituye al sujeto que lo habita. Lo que le hace falta de vez en cuando, es la resolución de leerlo y, ojalá, trabajar para cambiarlo.

\section{Referencias}

BERLIN, James A. Rhetoric and ideology in the writing class. College English, Vol. 50, no. 5, p. 477-494, 1988.

CORCORAN, Lucas. "Languaging 101": Translingual practices for the translingual realities of the SEEK composition clasroom. Journal of Basic Writing, vol. 36, no. 2, p. 54-77, 2017.

Bourdieu, Pierre. Le sens pratique. Paris: Editions de Minuit, 1980.

FOUCAULT, Michel. La historia de la sexualidad: La voluntad de saber. Traducción Ulises Guiñazú. México: Siglo XXI Editores, 1977.

FLORES, Nelson y ROSA Jonathan. Undoing appropriateness: Raciolinguistic ideologies and language diversity in education. Harvard Educational Review, vol. 86, no. 2, p. 149-171, 2015.

FULLINWIDER, Robert K. y LICHTENBURG, Judith. Leveling the playing field: Justice, politics and college admissions. Oxford: Rowman \& Littlefield Publishers, 2004.

FREIRE, Paulo. Pedagogía del oprimido. Traducción Jorge Mellado. México: Siglo XXI Editores, 1970.

MATSUDA, Paul Kei. The myth of linguistic homogeneity in U.S. college composition. College English, vol. 68, no. 6, p. 637-651, 2006.

Kögler, Hans-Herbert. The power of dialogue: Critical hermeneutics after Gadamer and Foucault. Traducción Paul Hendrickson. Boston: MIT Press, 1996. 
OTHEGUY Ricardo, GARCÍA Ofelia y REID Wallis. Clarifying translanguaging and deconstructing named languages: A perspective from linguistics. Applied Linguistics Review, vol. 6, no. 3, p. 281-307, 2015.

PENNYCOOK, Alastair. Language as a local practice. Oxford: Routledge, 2010.

PRATT, Mary Louis. Arts of the contact zone. Profession, p. 33-40, 1991.

SHOR, Ira. Critical teaching and everyday life. Boston: South End Press, 1980.

SHOR, Ira. Empowering education. Chicago: University of Chicago Press, 1992.

VERTOVEC, Steven. Super-diversity and its implicactions. Ethnic and Racial Studies, vol. 30, no. 6, p. 1024-1054, 2007.

Recebido em: 30/04/2019 Aprovado em: 31/10/2019 Publicado em: 20/12/2019 